Check for updates

Cite this: Soft Matter, 2017, 13,8113

Received 28th July 2017, Accepted 30th October 2017

DOI: $10.1039 / \mathrm{c} 7 \mathrm{sm} 01504 \mathrm{f}$

rsc.li/soft-matter-journal

\title{
Active Brownian equation of state: metastability and phase coexistence $\dagger$
}

\author{
Demian Levis, (D) *ab Joan Codina ${ }^{\mathrm{ab}}$ and Ignacio Pagonabarraga ${ }^{\mathrm{abc}}$
}

\begin{abstract}
As a result of the competition between self-propulsion and excluded volume interactions, purely repulsive self-propelled spherical particles undergo a motility-induced phase separation (MIPS). We carry out a systematic computational study, considering several interaction potentials, systems confined by hard walls or with periodic boundary conditions, and different initial conditions. This approach allows us to identify that, despite its non-equilibrium nature, the equations of state of Active Brownian Particles (ABP) across MIPS verify the characteristic properties of first-order liquid-gas phase transitions, meaning, equality of pressure of the coexisting phases once a nucleation barrier has been overcome and, in the opposite case, hysteresis around the transition as long as the system remains in the metastable region. Our results show that the equations of state of ABPs account for their phase behaviour, providing a firm basis to describe MIPS as an equilibrium-like phase transition.
\end{abstract}

\section{Introduction}

Active matter made of self-propelled particles can be found in a wide variety of contexts, both in living and synthetic systems, like bacteria or colloidal artificial swimmers. ${ }^{1}$ The fundamental difference between active and 'passive' matter made of thermally agitated constituents, is that the microscopic dissipative dynamics of the former breaks detailed balance and, as such, evolves far-from-equilibrium. The intrinsic out-of-equilibrium nature of active matter manifests strikingly in the presence of interactions. Self-propelled particles accumulate in regions of space where their velocity decreases as a consequence of collisions (between particles or with external obstacles). Simple models that capture in a minimal way the competition between selfpropulsion and steric effects, like the so-called Active Brownian Particles (ABP) model, have provided much insight into the generic behavior of such systems. For instance, at high enough densities and activities, a purely Motility-Induced Phase Separation (MIPS) generically takes place, leading to the coexistence of an active low density gas with a high density drop in the absence of attractive forces. ${ }^{2-7}$ This out-of-equilibrium transition is reminiscent of equilibrium liquid-gas de-mixing. It is thus tempting to extend the thermodynamic description of first order phase transitions in terms of, for instance, equations of

\footnotetext{
${ }^{a}$ Departament de Física de la Matèria Condensada, Universitat de Barcelona, Martí i Franquès 1, E08028 Barcelona, Spain.E-mail: demianparatodos@gmail.com

${ }^{b}$ University of Barcelona Institute of Complex Systems (UBICS), Universitat de Barcelona, Barcelona, Spain

${ }^{c}$ CECAM Centre Européen de Calcul Atomique et Moléculaire, École Polytechnique Fédérale de Lausanne, Batochimie, Avenue Forel 2, 1015 Lausanne, Switzerland

$\dagger$ Electronic supplementary information (ESI) available. See DOI: 10.1039/c7sm01504f
}

state, to ABP. However, this poses several fundamental difficulties since no thermodynamic variable is, in principle, well defined in this context. Much effort has been recently devoted to this question: the notions of effective temperature ${ }^{8-12}$ and chemical potential ${ }^{13}$ have been introduced, and special attention has been paid to the notion of pressure. ${ }^{14-21}$

In spite of the above-mentioned efforts, the equality of pressure at coexistence imposed by the very existence of an equation of state (EoS) for spherical $\mathrm{ABP},{ }^{17}$ has not been properly confirmed neither by experiments nor simulations. ${ }^{15,17-20}$ Several numerical studies have measured the pressure in ABP, but its interpretation has raised several conceptual problems. For instance, an abrupt pressure drop at the vicinity of MIPS has been reported and it has been argued that it constitutes a distinctive feature of this transition, hindering the analogy with equilibrium phase separation. ${ }^{19,20}$ A pressure loop generically appears in phase separating finite systems in equilibrium, an effect that can be redressed by the Maxwell construction. However, this construction is violated for $\mathrm{ABP},{ }^{17,22}$ so there is no direct way to understand the phase behaviour of the system from the simulated EoS. The phase diagram of ABP has been discussed in terms of binodal and spinodal-like curves. ${ }^{5,23}$ While the phase separation kinetics show the hallmarks of nucleation and spinodal decomposition, ${ }^{5,6,24,25}$ the connection with (static) quantities like the EoS remains unclear. Thus, the possibility to construct from the EoS a thermodynamic description of MIPS, bridging the gap between the phase diagram, the relaxation dynamics and the pressure, is still a matter of debate.

In this work we clarify these issues, providing a full characterization of the phase behavior of spherical ABPs in terms of its EoS and show that MIPS is fully consistent with the 
first-order phase transition scenario. We bring out the existence of a metastability region: an hysteresis around the coexistence pressure is found as the system is quenched to the coexistence region from 'below' or 'above' MIPS. As we show, the pressure drop near MIPS disappears as the system is quenched from 'above', and we recover instead a flatter EoS between the binodals, showing the equality of pressure at coexistence and thus allowing to estimate the location of the low-density binodal from the extrapolation of the pressure measurements above the spinodal. We analyze both open and confined systems in order to establish the role played by the presence of a nucleation barrier in the pressure anomalies reported in previous numerical works, and the inherent difficulty of sampling the thermodynamic behaviour of the EoS of ABPs at coexistence. We find that the EoS of an open system quenched from 'above' approaches the behaviour of the system under confinement, showing that the difficulties in interpreting the pressure data are due to the presence of a large nucleation barrier that can be easily bypassed by including a nucleation core (a wall). Consistently, as we show, MIPS can be stabilized at much lower densities (between the binodal and spinodal line) than those reported to date, and the phase diagram can be understood from the EoS despite the absence of a Maxwell construction.

In order to show the generality of our results, we perform NVT simulations of spherical ABPs using different repulsive potentials. As we show, the location and depth of the pressure drop found for different potentials can be rationalized borrowing ideas from classical nucleation theory. Thus, we connect the structure of the dense phase and its phase separation kinetics: stiffer potentials promote the emergence of crystalline ordering accompanied by a large surface tension, explaining the presence of a large nucleation barrier and, therefore, the difficulties that have hindered the interpretation of the EoS of ABPs.

\section{Model}

To be specific, we consider $N$ self-propelled particles in a $2 d$ volume $V=L_{x} \times L_{y}$. The model is defined by the following equations of motion for each particle at position $\mathbf{r}_{i}=\left(x_{i}, y_{i}\right)$ and with orientation $\mathbf{n}_{i}(t)=\left(\cos \theta_{i}, \sin \theta_{i}\right)$ :

$$
\dot{\mathbf{r}}_{i}=v_{0} \mathbf{n}_{i}+\mu \mathbf{F}_{i}+\sqrt{2 D_{0}} \xi_{i}, \dot{\theta}_{i}=\sqrt{2 D_{\theta}} \nu_{i}
$$

where $\xi_{i}$ and $\nu_{i}$ are zero-mean unit-variance Gaussian noises, and $v_{0}$ is a constant self-propulsion velocity. The force $\mathbf{F}_{i}$ is the sum of inter-particle interactions, $\mathbf{F}_{i}^{\text {int }}=-\frac{1}{2} \nabla_{i} \sum_{j \neq i} u\left(r_{i j}\right)=$ $\frac{1}{2} \sum_{j \neq i} \mathbf{f}_{i j}$ and external potentials, $\mathbf{F}_{i}^{\text {ext }}=-\nabla_{i} w\left(r_{i}\right)$. Here we compare Active Brownian (infinitely) Hard Disks (AB-HD)

$$
u(r)=\left\{\begin{array}{cc}
\infty & r<\sigma \\
0 & r \geq \sigma,
\end{array}\right.
$$

with particles interacting with a Weeks-Chandler-Andersen potential (AB-WCAD)

$$
u(r)=\left\{\begin{array}{ll}
4 \varepsilon\left[\left(\frac{\sigma}{r}\right)^{12}-\left(\frac{\sigma}{r}\right)^{6}\right]+\varepsilon & r<2^{1 / 6} \sigma \\
0 & r \geq 2^{1 / 6} \sigma
\end{array},\right.
$$

with a characteristic energy scale $\varepsilon$. Contrary to ref. 23, these potentials diverge at the origin, setting a natural bound to the infinite softness limit at high $v_{0}$. In order to analyze the impact of confinement on the equations of state, we use an external potential along the $x$-axis: $\mathbf{F}^{\text {ext }}\left(\mathbf{r}_{i}\right)=-\nabla_{i} w\left(x_{i}\right)$ where

$$
w(x)=\left[x^{-12}+\left(L_{x}-x\right)^{-12}\right] \text {. }
$$

The units of length and time are given by $\sigma$ and $D_{\theta}{ }^{-1}$, respectively, and fix $D_{\theta}=3 D_{0} / \sigma^{2}$. The phase behavior of the model (for both $\mathrm{AB}-\mathrm{HD}$ and $\mathrm{AB}-\mathrm{WCAD}$ ) is controlled by the following non-dimensional parameters: the packing fraction $\phi=\pi \sigma^{2} N /(4 V)$, which quantifies crowding effects, the Péclet number, Pe $=v_{0} /\left(\sigma D_{\theta}\right)$, which quantifies the strength of selfpropulsion, and the effective particle stiffness $\Gamma=\varepsilon \mu /\left(\sigma v_{0}\right)$, which naturally arises from the inter-particle energy scale $\varepsilon$. This parameter, which quantifies to what extent particles become effectively softer as their activity increases, is irrelevant in the limit of infinitely hard-disks.

We carry out Brownian Dynamics (BD) simulations to study the evolution of the system in the absence of any external forcing. Since it is not straightforward to deal with the singular nature of hard core interactions in BD simulations, we use a variant of Event-Driven Brownian Dynamics (ED-BD) for hard spheres $^{26}$ that accounts for self-propulsion. $\neq^{27}$ We simulated systems of $N=2000, \ldots, 8000$ AB-HD using a Brownian time step $\tau_{\mathrm{B}}=0.01$ which fixes $D_{0}=\tau_{\mathrm{B}} / 2=0.005$ and thus $D_{\theta}=0.015$. Self-propulsion in the ED-BD scheme is introduced in such a way that the collisions are elastic and do not modify the orientation $\mathbf{n}_{i}$ of the particles. We vary the Peclet number Pe by varying $v_{0}$, from $v_{0}=0(\mathrm{Pe}=0)$ to $v_{0}=1.5(\mathrm{Pe}=100)$. For AB-WCAD, we use a second order stochastic Runge-Kutta algorithm as described in ref. 28 with a time step $\Delta t=10^{-5}$, $5 \times 10^{-5}$ (used to simulate systems with the highest and the lowest Pe numbers explored, respectively). To analyze the impact of the stiffness of the potential, we simulated systems with $\varepsilon=0.4$ up to $\varepsilon=250$.

\section{Phase diagram}

The binodal-like curves $\left(\phi_{\text {low }}, \phi_{\text {high }}\right)$ shown in Fig. 1 can be readily constructed from the coexisting densities. ABP with different stiffness exhibit the same qualitative phase behavior: above a critical Pe they undergo MIPS if its average density is large enough.

$\ddagger$ The constraint that two particles cannot overlap can also be implemented by a Monte Carlo (MC) procedure. ${ }^{7}$ The MC dynamics gives rise to clusters with selflimiting size, ${ }^{7}$ while ED-BD produces clusters that coarsen by MIPS. 

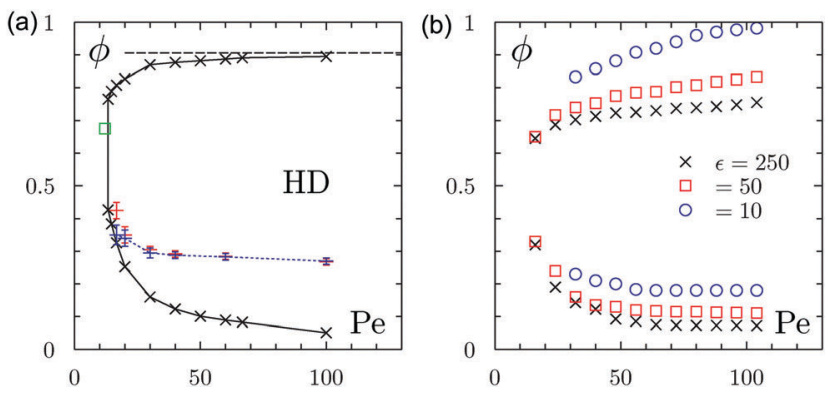

Fig. $1 \mathrm{Pe}-\phi$ phase diagram of ABP using PBC. Left: AB-HD. Black symbols show the coexisting densities $\phi_{\text {low }}$ and $\phi_{\text {high }}$ defining the binodals. Red points with errorbars indicate the onset of MIPS (see Fig. 2 for details). Blue points correspond to the location of the pressure drop. We identify a critical point at $\mathrm{Pe}_{\mathrm{c}} \approx 12$ and $\phi_{\mathrm{c}} \approx 0.674$ (in green). The horizontal dotted line indicates the closest packing of hard disks $\phi_{\mathrm{cp}}=\pi /(2 \sqrt{3})$. Right: AB-WCAD for different stiffness. The symbols show the two binodals $\phi_{\text {low }}$ and $\phi_{\text {high }}$

To identify MIPS for AB-HD with PBC we let the system evolve from an homogeneous initial condition and then, once the system has reached its steady-state, we measure the fraction of particles in the dense phase $\Psi$. We compute $\Psi$ as the probability that a given particle belongs to the largest cluster in the system, where a cluster is defined as a connected set of neighboring particles (defined by a distance $1.05 \sigma$ ). In order to locate more precisely the appearance of MIPS we also compute its associated second moment

$$
\chi=N\left(\left\langle\Psi^{2}\right\rangle-\langle\Psi\rangle^{2}\right)
$$

As shown in Fig. 2(a) this quantity grows from $\Psi \approx 0$ to $0<\Psi \lesssim 1$ as we increase the packing fraction at fixed Pe. The corresponding susceptibility $\chi$ shows a peak at the onset of MIPS (Fig. 2(b)), thus providing a criterion to locate it in the $\mathrm{Pe}-\phi$ phase diagram. The transition points $\phi_{\mathrm{n}}$ obtained using this prescription are shown by red symbols in the phase diagram Fig. 1(a). As it is clear from the figure, these transition points remain rather close to the location of the pressure discontinuity (see Section 4), shown by blue symbols. As discussed below, the location of the pressure discontinuity results in a
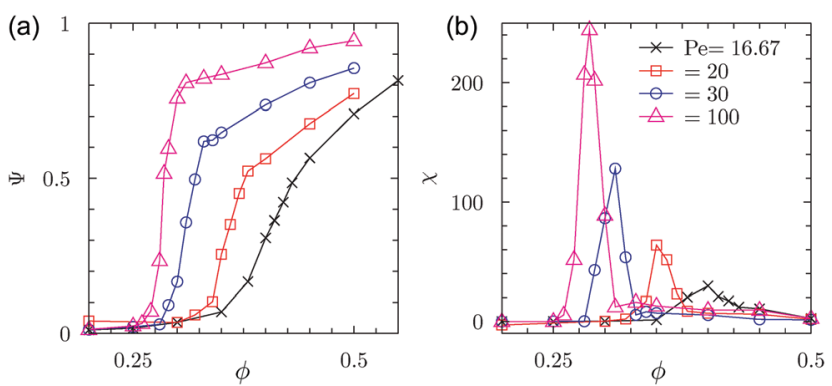

Fig. 2 Left: Probability of a given particle to belong to the largest cluster $\Psi$ as a function of the packing fraction for several Pe values. Right: Corresponding reduced second moment of $\Psi, \chi$, as a function of $\phi$. All the data shown here is the result of 100 independent simulations of $N=4000 \mathrm{AB}-\mathrm{HD}$ using $\mathrm{PBC}$ and a homogeneous disordered state as initial condition. spinodal-like curve, as shown in Fig. 1(a) by a blue dotted line, and as shown by our cluster analysis, it is accompanied by the emergence of a macroscopic cluster.

To understand the role of particle stiffness, we construct the binodals for AB-WCAD (see Fig. 1(b)). Increasing $\varepsilon$ one observes both a decrease of $\phi_{\text {low }}$ and an increase in the structure, as depicted in Fig. 5(d). This feature implies that the high-density phase has a higher degree of order for stiffer potentials. In the limit of hard disks, the high-density branch of the binodal, $\phi_{\text {high }}$, quickly saturates at close packing, indicating that the competition between self-propulsion and excluded volume generates a nearly perfect hexagonal crystal at high activities, as shown in Fig. 5 (e.g. for $\mathrm{Pe}=60$, a dense region at $\phi_{\text {high }}=0.98 \phi_{\mathrm{cp}}$ coexists with a gas at $\phi_{\text {low }}=0.089, \phi_{\mathrm{cp}}=\pi /(2 \sqrt{3})$ is the closest packing of disks).

\section{Equation of state}

We identify the pressure, $P$, by an extension of the virial theorem that accounts for active forces: ${ }^{19,29}$

$$
P=\rho k_{\mathrm{B}} T+P_{\text {int }}+P_{\mathrm{a}}
$$

where $\rho k_{\mathrm{B}} T$ is the ideal gas pressure,

$$
P_{\text {int }}=\frac{1}{4 V} \sum_{i} \sum_{j}\left\langle\mathbf{f}_{i j} \cdot\left(\mathbf{r}_{i}-\mathbf{r}_{j}\right)\right\rangle
$$

is the standard virial expression of the collisional pressure, and

$$
P_{\mathrm{a}}=\frac{v_{0}}{2 \mu V} \sum_{i}\left\langle\mathbf{n}_{i} \cdot \mathbf{r}_{i}\right\rangle=\frac{\rho v_{0} v_{\phi}}{2 \mu D_{\theta}}, v_{\phi}=\frac{1}{N} \sum_{i}\left\langle\mathbf{n}_{i} \cdot \dot{\mathbf{r}}_{i}\right\rangle
$$

is the active contribution to the pressure or swim pressure. ${ }^{15,16,30}$ The total pressure $P$ defined this way is a state function for spherical $\mathrm{ABP}^{17}$ and its definition can be extended to unconfined systems where $P$ is equal to the internal bulk pressure. ${ }^{19}$

Following usual practice, we study the phase behavior of $\mathrm{ABP}$ by letting the system evolve from a homogenous initial state at some density $\phi$ towards its stationary state corresponding to a given value of Pe. This procedure corresponds to a quench from $\mathrm{Pe}=0$ to $\mathrm{Pe}>0$. In order to gain insight into the nature of the pressure, we compare the values obtained for a periodic system and one confined in the $x$-direction by two walls at $x=0$ and $x=L_{x}$, and periodic boundary conditions (PBC) in the $y$-direction.

The $P(\phi ; \mathrm{Pe})$ equations of state (normalized by the ideal gas pressure $P_{0}$ and multiplied by $\phi$ ) found in confined and open geometries are shown in Fig. 3. If the system does not phase separate, for $\mathrm{Pe}<\mathrm{Pe}_{\mathrm{c}}$, the total pressure of $\mathrm{AB}-\mathrm{HD}$ with $\mathrm{PBC}$ coincides with the pressure computed in confined conditions (see Fig. 3(a)). At higher activities, for PBC the pressure drops abruptly in the vicinity of MIPS, ${ }^{19,20}$ while in the confined system it displays a monotonic increase with density and remains roughly constant in the coexistence region. As shown in Fig. 3(a and b), the pressure jump becomes more pronounced as the activity and the stiffness of the particles increases (note the similarity between $\mathrm{AB}-\mathrm{HD}$ with $\mathrm{Pe}=30$ and $\mathrm{AB}-\mathrm{WCAD}$ 

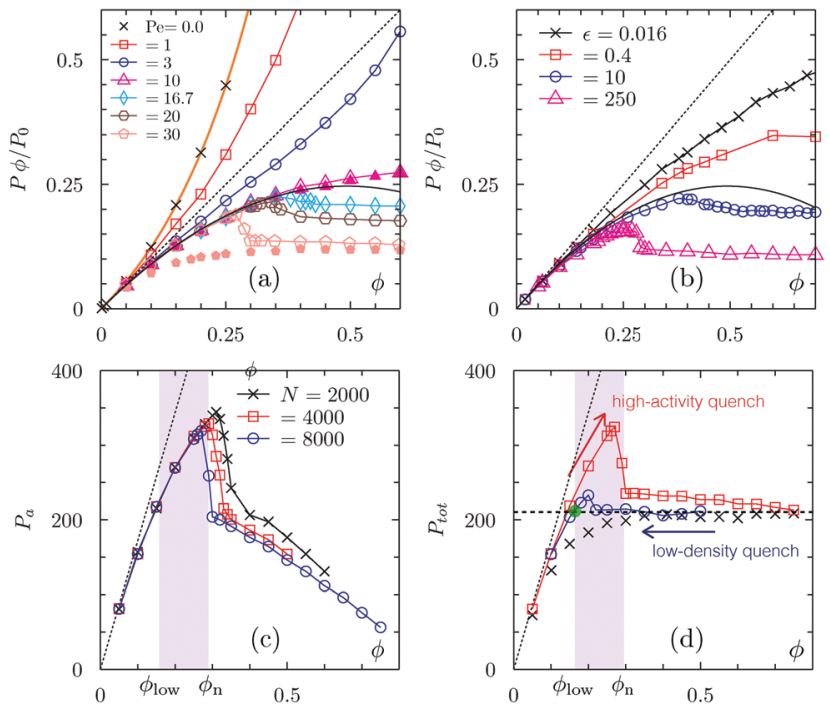

Fig. 3 (a) Total pressure times $\phi$ normalized by the ideal gas pressure $P_{0}$ of $\mathrm{AB}-\mathrm{HDs}$ for $\mathrm{Pe}=0, \ldots, 30$ in systems with PBC (line-open symbols) and for $\mathrm{Pe}=10$ and 30 in systems with confining walls (in filled triangular and pentagonal symbols, respectively). The continuous (orange) line corresponds to the analytical equation of state for hard disks; ${ }^{31}$ the dotted one is the ideal gas limit. The black curve shows $P / P_{0}=1-\phi$. (b) Total pressure of $A B-W C A D s$ using $P B C$ and different stiffness at fixed $P e=100$. The continuous and dotted lines are the same as in (a). (c) Active pressure for systems of $A B-H D s$ with PBC (line-points) and confining walls (symbols) of different size for $\mathrm{Pe}=30$. (d) Hysteresis in the EoS of $\mathrm{AB}-\mathrm{HDs}$ for $\mathrm{Pe}=30$ : the red points are obtained by quenching the system with PBC from an homogenous state; the blue points were obtained by quenching the system with $\mathrm{PBC}$ from a phase separated state (at $\phi=0.50, \mathrm{Pe}=30$ ). Black points show the equation of state in the confined system. The coexistence pressure $P^{*}$ is represented by a flat broken-line and its intersection with the low density branch of the equation of state of the open system (blue dots) is shown by a green symbol. The light colored band in panels (c and d) shows the metastable region between the binodal $\phi_{\text {low }}$ and the onset of MIPS $\phi_{n}$.

with $\varepsilon=250$ and $\mathrm{Pe}=100$ ). The formation of a macroscopic cluster, due to MIPS, coincides with the location of the pressure drop, $\phi_{\mathrm{n}}$ (as discussed above). Particles are dramatically slowed down as they aggregate, making $v_{\phi}$, and therefore the active pressure, to drop (see eqn (6)). The low-density branch of the binodal, $\phi_{\text {low }}$, is well below the density $\phi_{\mathrm{n}}$ at which the dense phase appears.

Above $\mathrm{Pe}_{\mathrm{c}}$, but for densities below the onset of MIPS, the equations of state collapse into a single curve which can be fitted by a first order virial expansion, $P=P_{0}\left(1+b_{1} \phi\right)$, where $P_{0}$ is the pressure in the ideal gas limit. ${ }^{19}$ The equations of state (or compressibility factor) of AB-HD in confined conditions are shown in Fig. 4. Together with the data points, we also show the Carnahan-Starling equation of state for passive Hard-Disks ${ }^{31}$ and the best fits of the simulation data at Pe $>0$ by a second order virial expansion

$$
P / P_{0}=1+b_{1} \phi+b_{2} \phi^{2} .
$$

As shown in Fig. 4, in confined conditions, $b_{1}$ decreases monotonically with $\mathrm{Pe}$, in consistency with the viewpoint that self-propulsion induces an effective attraction..$^{2,12,18}$ Surprisingly, for non-confined systems, $b_{1}$ is independent of Pe once MIPS has

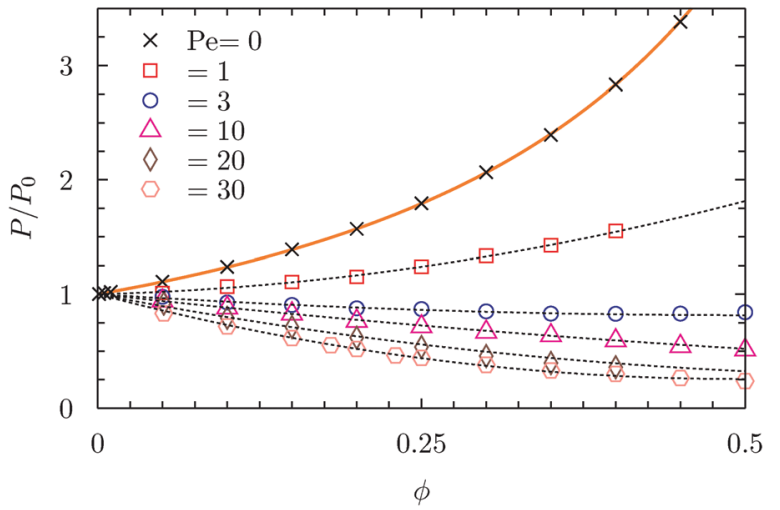

Fig. 4 Equations of state of $\mathrm{AB}-\mathrm{HD}$ confined along one direction between two hard walls for several Pe numbers. Dotted lines denote fits by a second order virial expansion. The continuous line is the CarnahanStarling equation of state for Hard-Disks. ${ }^{31}$

taken place, indicating that the activity-induced attraction saturates above $\mathrm{Pe}_{\mathrm{c}}$ (as reported in ref. 17, 19 and 20). As we show below, this is due to the fact that the open system evolves along a metastable branch and remains in its low-density phase above $\phi_{\text {low }}$, until it reaches $\phi_{\mathrm{n}}$, when a dense cluster emerges by MIPS.

Interestingly, the equations of state in confined and PBC geometries converge at large densities to a value close to the expected coexistence pressure. In equilibrium, finite-size systems display a pressure loop due to the formation of an interface between the two phases, ${ }^{32,33}$ while the loop arising in van der Waals (mean-field) theory is (thermodynamically) unstable. Accordingly, $P(\phi)$ shows a peak when the dense phase develops, an effect that should disappear in the thermodynamic limit. In simulations, this behavior is typically smoothed out by interface fluctuations. The sharp decrease in $P$ for ABPs implies that interfacial fluctuations are considerably suppressed, suggesting a large interface tension (indeed AB-HD generate an interface between a dilute gas and a closed packed crystal). Hence, we can interpret the pressure drop for PBC systems as a finite size effect. For finite systems the EoS is affected both because of the interfacial contribution and a shift in the location of nucleation.§ These two effects are consistent with the data shown in Fig. 3(c). The active pressure in systems of $N \gtrsim 4000$ converges above $\phi_{\mathrm{n}}$, meaning that there is no interfacial contribution to the pressure after phase separation. It is less clear though whether the pressure jump is reduced when increasing $N_{.}{ }^{20}$ We push thermodynamic ideas even further, and claim that the system sizes used so far are not large enough to observe how the onset of MIPS approaches the binodal. The idea behind this claim is that MIPS involves a large critical nucleus: our systems turn out to be too small to phase separate close to the binodal and thus remain metastable in a sub-region of the coexistence region. $\uparrow$ The convergence of $\phi_{\mathrm{n}}$ towards the binodal as the system is made larger is due to the fact that the probability to spontaneously

$\S$ For a first-order phase transition in equilibrium, the interface free energy density vanishes as $\Delta f \sim 1 / \sqrt{N}$ and the nucleation point is shifted by $\phi_{\mathrm{n}} \sim N^{-1 / 3} \cdot 32$

T Small systems should rather be thought in terms of clusters as discussed in ref. $34-36$. 
form a cluster of size $m>m_{\mathrm{c}}$, (where $m_{\mathrm{c}}$ is the critical nucleus size) increases with system size. The system sizes used need to be much larger than $m_{\mathrm{c}}$, otherwise, nucleation cannot take place and the approach of $P(\phi)$ to its infinite-size behavior cannot be observed. In the following we show this important claim.

\section{Metastability}

In order to probe metastability, we initialize the open system deeply in the coexistence region. As we expand the system, nucleation is avoided since an interface between a dense and low density phase is already present. We perform such a low- $\phi$ quench at fixed Pe by letting AB-HDs evolve from a steady-state at $\phi=0.50, \mathrm{Pe}=30$ to lower packing fractions. The equation of state we obtain is depicted in blue in Fig. 3(d). We find that: (i) the system remains phase separated at much lower densities than the ones previously reported (see Fig. 5(a) and movie in ref. 37); (ii) the pressure remains roughly constant down to $\phi \approx \phi_{\text {low }}$. We thus found hysteresis around MIPS, a typical signature of first-order phase transitions.

Accordingly to classical nucleation theory (CNT), in the absence of a preferential site (homogeneous nucleation), phase separation can only be triggered by a rare event: the spontaneous formation of a critical nucleus of size larger than $m_{\mathrm{c}} \propto \gamma / \Delta G_{\text {homo }}$ (where $\gamma$ is the interface tension and $\Delta G_{\text {homo }}$ the free energy difference). Since our system is out-of-equilibrium, CNT cannot be directly applied. However, borrowing ideas from equilibrium systems, Redner et al. have developed an analogous theory to describe the kinetics of phase separation in $\mathrm{ABP},{ }^{24}$ providing a theoretical description of simulation results. ${ }^{25}$ Within this framework, $m_{\mathrm{c}} \propto \phi_{\mathrm{cp}} / \ln ^{2}\left(\mathrm{Pe} \phi_{\text {low }}\right)$. To make a comparison with simulations, an expression $\phi\left(A, \phi_{\text {high }}, \phi_{\text {low }}\right)$ for the average packing fraction in terms of the theoretical predictions is established, where $A$ is completely determined by the cluster size distribution $\mathscr{P}_{\mathrm{m}}$ (see eqn (11) in ref. 24). The nucleation barrier is thus controlled by two main ingredients: the location of the binodals and the structure of the clusters. We computed $\mathscr{P}_{\mathrm{m}}$ for AB-HD and $\mathrm{AB}-\mathrm{WCAD}$ with different $\varepsilon$ and found a roughly identical distribution (see Fig. 5(c)). For stiffer potentials the structural difference between the coexisting phases is more severe. In the hard-disk limit the binodals are pushed to their limit values $\phi_{\text {low }} \rightarrow 0$ and $\phi_{\text {high }} \rightarrow \phi_{\text {cp }}$ as the activity increases. Therefore, $m_{\mathrm{c}}$ is expected to be very large at high Pe. As shown in Fig. 5(d), softening the particles suppresses the order of the dense phase. In turn, $\phi_{\text {low }}$ is larger for softer potentials (thus $m_{\mathrm{c}}$ is smaller), qualitatively explaining the reduction of the pressure drop for softer disks in Fig. 3(b). $\|$ We did not intend to make a direct quantitative comparison between theory and simulations, but, at this level, we are able to insure that for hard ABPs, MIPS features a large nucleation barrier that discourages attempts to observe how the system escapes from metastability with BD simulations.

$\|$ Redner et al. predict $m_{\mathrm{c}} \approx 5000$ at $\mathrm{Pe}=30$ and $\phi=0.30$ for AB-WCAD with $\varepsilon=1$ in our units (see ref. 24). This value lies quite close to the binodal in their case while for $\mathrm{AB}-\mathrm{HD}$ it falls deep into the coexistence region.
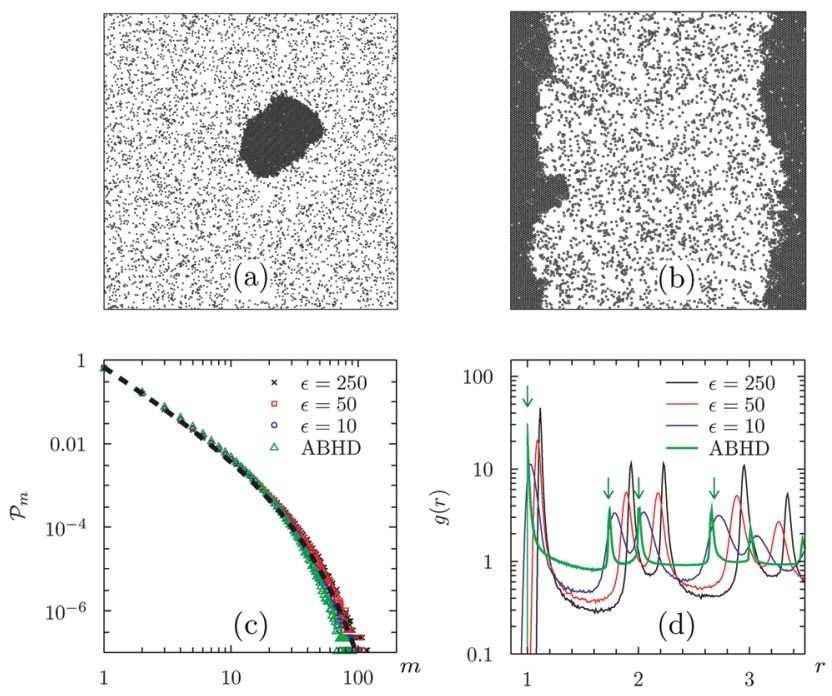

Fig. 5 (a) Snapshot of an open system made of $N=8000$ AB-HD with PBC at $\phi=0.12$ and $P e=60$ obtained from a low- $\phi$ quench. (b) Snapshot of $N=8000 \mathrm{AB}-\mathrm{HD}$ in the confined geometry for $\phi=0.30$ and $\mathrm{Pe}=30$. (c) Cluster size distribution for ABP, both AB-HD $(\varepsilon \rightarrow \infty)$ and AB-WCAD, with $\mathrm{PBC}$ at $\phi=0.2, \mathrm{Pe}=30$ and several stiffness. For comparison, we show in dotted lines: $\propto \exp \left(-\mathrm{m} / \mathrm{m}^{\star}\right) / \mathrm{m}^{2}$ with $m^{*}=14.5$. (d) Pair correlation function at fixed $\mathrm{Pe}=30$ and $\phi=0.35$ for several potentials. The arrows indicate $r=1, \sqrt{3}, 2$ and $1+\sqrt{3}$ corresponding to an hexagonal packing.

To give further support to this idea we turn now our attention into the system in presence of hard walls.

Confinement facilitates nucleation because of wetting. As shown in Fig. 5(b), and discuss previously in the literature, ABP accumulate at walls which thus act as natural nucleation seeds. ${ }^{12,21,23}$ The free energy associated with (heterogeneous) nucleation in a confined system is

$$
\Delta G_{\text {het }}=F(\alpha) \Delta G_{\text {homo }}
$$

where

$$
F(\alpha)=(2+\cos \alpha)(1-\cos \alpha)^{2} / 4
$$

is a function of the contact angle $\alpha$ between the wall and the dense phase. The adsorption of ABP into layers give $\alpha=0$ (pure wetting) and therefore, by extension of CNT, a vanishing nucleation barrier $\Delta G_{\text {het }}=0$. This equilibrium-like description is consistent with the absence of a pressure drop in the presence of walls and confirms our overall interpretation of the EoS across MIPS.

Consistently, the value of the pressure obtained in the open system with PBC (using both a low- $\phi$ or high- $\phi$ quench), and in confinement, converges to the same constant value far away from the spinodal, thus defining the coexistence pressure $P^{*}$. As shown by the discontinuous flat line in Fig. 3(d), the extrapolation of $P^{*}$ across the metastability region provides an independent numerical estimation of the location of the binodal, $\phi_{\text {low }}{ }^{*}$ (shown by a green symbol), defined as the packing fraction at which the low density branch of the $\operatorname{EoS}\left(\phi<\phi_{\mathrm{n}}\right)$ intersects $P^{*}$. This 'data-based' construction provides the (approximate) location of the binodal from the pressure measurements, in agreement 
with the density distribution; thus connecting the EoS obtained from simulations and the coexistence phase diagram.

\section{Conclusion}

We have carefully examined the pressure of ABPs using different potentials, topologies and initial conditions in order to show that the EoS are fully consistent with the classical (equilibrium) first-order phase transition scenario. The equations of state of ABP do not exhibit any fundamental difference to an equilibrium system showing phase coexistence, besides: (i) the absence of a Maxwell construction on $P$; (ii) the extreme structural difference between the two coexisting phases, giving rise to a large nucleation barrier. Overall, our work results in a systematic way to interpret the equations of state of ABP using equilibrium-like concepts. We shed light into the first-order nature of MIPS, clarifying the analogies with liquid-gas phase separation and providing a consistent framework that brings together the phase separation kinetics, the coexistence density curves and the equations of state of spherical ABP.

We have focussed on the case of isotropic Active Brownian Particles (disks), for which an equation of state exists. The existence of a metastability region with a large nucleation barrier and the equality of pressure at coexistence has been brought out by the direct comparison between the pressure in a confined and periodic system. Of course, such an approach is meaningful as long as the pressure is a state function. However, this crucial property is quite fragile in active systems and it generically fails as soon as anisotropic interactions are involved. ${ }^{38,39}$ Shape anisotropy (active particles like bacteria are often elongated) might in turn affect the MIPS scenario, since orientational ordering has to be taken into account in these systems. ${ }^{40,41}$ Therefore, the characterization of the phase behavior of anisotropic self-propelled particles using thermodynamic-like ideas, as the ones we put forward here, presents a fundamental difficulty. The nature of phase transitions in this kind of systems is still unclear, thus calling for further developments.

\section{Conflicts of interest}

There are no conflicts to declare.

\section{Acknowledgements}

DL warmly thanks R. Ni, G. Redner, A. Solon, T. Speck, C. Wagner and T. Voigtmann for useful exchanges, and in particular J. Tailleur for his suggestions. DL acknowledges funding from a Marie Sklodowska-Curie Individual Fellowship (G.A. no. 657517). JC is supported by Spanish MECD fellowship FPU13/01911. IP acknowledges MINECO and DURSI for financial support under projects FIS2015-67837- P and 2014SGR-922, respectively.

\section{References}

1 M. C. Marchetti, J. F. Joanny, S. Ramaswamy, T. B. Liverpool, J. Prost, M. Rao and R. A. Simha, Rev. Mod. Phys., 2013, 85, 1143.

2 J. Tailleur and M. E. Cates, Phys. Rev. Lett., 2008, 100, 218103.

3 M. E. Cates and J. Tailleur, Annu. Rev. Condens. Matter Phys., 2015, 6, 219.

4 Y. Fily and M. C. Marchetti, Phys. Rev. Lett., 2012, 108, 235702.

5 G. S. Redner, M. F. Hagan and A. Baskaran, Phys. Rev. Lett., 2013, 110, 055701.

6 J. Stenhammar, D. Marenduzzo, R. J. Allen and M. E. Cates, Soft Matter, 2014, 10, 1489.

7 D. Levis and L. Berthier, Phys. Rev. E: Stat., Nonlinear, Soft Matter Phys., 2014, 89, 062301.

8 D. Loi, S. Mossa and L. F. Cugliandolo, Phys. Rev. E: Stat., Nonlinear, Soft Matter Phys., 2008, 77, 051111.

9 J. Palacci, C. Cottin-Bizonne, C. Ybert and L. Bocquet, Phys. Rev. Lett., 2010, 105, 088304.

10 G. Szamel, Phys. Rev. E: Stat., Nonlinear, Soft Matter Phys, 2014, 90, 012111.

11 D. Levis and L. Berthier, Europhys. Lett., 2015, 111, 60006.

12 U. M. B. Marconi and C. Maggi, Soft Matter, 2015, 11, 8768.

13 M. Dijkstra, S. Paliwal, J. Rodenburg and R. van Roij, arXiv preprint arXiv:1609.02773, 2016.

14 S. A. Mallory, A. Šarić, C. Valeriani and A. Cacciuto, Phys. Rev. E: Stat., Nonlinear, Soft Matter Phys., 2014, 89, 052303.

15 S. C. Takatori, W. Yan and J. F. Brady, Phys. Rev. Lett., 2014, 113, 028103.

16 X. Yang, M. L. Manning and M. C. Marchetti, Soft Matter, 2014, 10, 6477.

17 A. P. Solon, J. Stenhammar, R. Wittkowski, M. Kardar, Y. Kafri, M. E. Cates and J. Tailleur, Phys. Rev. Lett., 2015, 114, 198301.

18 F. Ginot, I. Theurkauff, D. Levis, C. Ybert, L. Bocquet, L. Berthier and C. Cottin-Bizonne, Phys. Rev. X, 2015, 5, 011004.

19 R. G. Winkler, A. Wysocki and G. Gompper, Soft Matter, 2015, 11, 6680.

20 A. Patch, D. Yllanes and M. C. Marchetti, Phys. Rev. E, 2017, 95, 012601.

21 T. Speck and R. L. Jack, Phys. Rev. E, 2016, 93, 062605.

22 A. P. Solon, J. Stenhammar, M. E. Cates, Y. Kafri and J. Tailleur, arXiv preprint arXiv:1609.03483, 2016.

23 Y. Fily, A. Baskaran and M. F. Hagan, Soft Matter, 2014, 10, 5609.

24 G. S. Redner, C. G. Wagner, A. Baskaran and M. F. Hagan, Phys. Rev. Lett., 2016, 117, 148002.

25 D. Richard, H. Löwen and T. Speck, Soft Matter, 2016, 12, 5257.

26 A. Scala, T. Voigtmann and C. De Michele, J. Chem. Phys., 2007, 126, 134109.

27 R. Ni, M. A. C. Stuart and M. Dijkstra, Nat. Commun., 2013, 4, 2704.

28 A. C. Branka and D. M. Heyes, Phys. Rev. E: Stat. Phys., Plasmas, Fluids, Relat. Interdiscip. Top., 1999, 60, 2381. 
29 G. Falasco, F. Baldovin, K. Kroy and M. Baiesi, New J. Phys., 2016, 18, 093043.

30 S. C. Takatori and J. F. Brady, Soft Matter, 2015, 11, 7920.

31 D. Henderson, Mol. Phys., 1975, 30, 971.

32 J. E. Mayer and W. W. Wood, J. Chem. Phys., 1965, 42, 4268.

33 M. Schrader, P. Virnau and K. Binder, Phys. Rev. E: Stat., Nonlinear, Soft Matter Phys., 2009, 79, 061104.

34 D. J. McGinty, J. Chem. Phys., 1973, 58, 4733.

35 J. K. Lee, J. A. Barker and F. F. Abraham, J. Chem. Phys., 1973, 58, 3166 .
36 D. Reguera, R. K. Bowles, Y. Djikaev and H. Reiss, J. Chem. Phys., 2003, 118, 340.

37 See ESI $\dagger$.

38 A. P. Solon, Y. Fily, A. Baskaran, M. E. Cates, Y. Kafri, M. Kardar and J. Tailleur, Nat. Phys., 2015, 11, 673-678.

39 M. Joyeux and E. Bertin, Phys. Rev. E, 2016, 93, 032605.

40 A. Suma, G. Gonnella, D. Marenduzzo and E. Orlandini, Europhys. Lett., 2014, 108, 56004.

41 S. Weitz, A. Deutsch and F. Peruani, Phys. Rev. E: Stat., Nonlinear, Soft Matter Phys., 2015, 92, 012322. 